\title{
The null energy condition and instability
}

\author{
Roman V. Buniy * Stephen D. H. Hsu周 and Brian M. Murray \\ Institute of Theoretical Science, University of Oregon, Eugene OR 94703-5203
}

\begin{abstract}
We extend previous work showing that violation of the null energy condition implies instability in a broad class of models, including gauge theories with scalar and fermionic matter as well as any perfect fluid. Simple examples are given to illustrate these results. The role of causality in our results is discussed. Finally, we extend the fluid results to more general systems in thermal equilibrium. When applied to the dark energy, our results imply that $w=p / \rho$ is unlikely to be less than -1 .
\end{abstract}

\section{INTRODUCTION}

Every spacetime is a possible solution to Einstein's equations for some particular choice of energymomentum tensor $T_{\mu \nu}$. It is therefore important to understand what general restrictions can be placed on the energy-momentum of a physical system. Such restrictions are referred to as energy conditions, and play an important role in general relativity. In an earlier paper 1], two of the present authors demonstrated a direct connection between stability and the null energy condition (NEC) [2], $T_{\mu \nu} n^{\mu} n^{\nu} \geq 0$ for any null vector $n^{\mu}$ (satisfying $\left.g_{\mu \nu} n^{\mu} n^{\nu}=0\right)$. The main results of the study in Ref. 1] are: (1) classical solutions of both minimally and non-minimally coupled scalar-gauge models which violate the NEC are unstable, (2) a quantum state in which the expectation of the energy-momentum tensor violates the NEC cannot be the ground state (includes models with fermions), (3) perfect fluids which violate the NEC are unstable. These results suggest that violations of the NEC in physically interesting cases are likely to be ephemeral.

Using the Raychaudhuri equation for the expansion of a hypersurface null congruence, one can show that null geodesics have non-increasing expansion as long as the NEC is obeyed [3]. This result plays an important role in the classical singularity theorems of general relativity [3] and in proposed covariant entropy bounds 4]. It also implies that NEC violating matter is necessary for the construction of Lorentzian wormholes [5].

This work is an extension of Ref. 1]. We give some explicit examples of the classical stability analysis (Sec. III), additional details regarding the local instability in quantum states which violate the NEC (Sec. III), a simplified and more general proof of the result that the fermionic effective action does not lead to violation of the NEC (Sec. IV], and a generalization of our analysis of fluids to any system in thermal equilibrium (Sec. V]. In Ref. [6] it was shown that models with superluminal modes can evade our results in some cases. However, in Ref. 7] it

\footnotetext{
*Electronic address: roman@uoregon.edu

${ }^{\dagger}$ Electronic address: hsu@duende.uoregon.edu

‡Electronic address: bmurray1@uoregon.edu
}

was noted that such models are acausal. In the appendix, we show that in causal models, the NEC is sufficient to determine stability. In Sec. III it is shown that considerations involving causality or superluminality are necessary only in cases where the classical background violates all rotational symmetries. Cosmological models of the dark energy must exhibit isotropy and hence are covered by the earlier results of Ref. 1].

The NEC has direct implications for the dark energy equation of state, often given in terms of $w=p / \rho$. Dark energy has positive energy density $\rho$ and the energymomentum tensor is $T_{\mu \nu}=\operatorname{diag}(\rho, p, p, p)$ in the comoving cosmological frame. Therefore, $w<-1$ implies violation of the NEC. Instability as a consequence of $w<-1$ was studied previously in scalar models 8] and in general in Ref. 1]. The analysis applies to dark energy models such as k-essence 9], rolling tachyon condensate 10], and phantoms [1].

\section{CLASSICAL FIELD THEORIES}

In this section we will first look at some examples and then derive the general result of Ref. 1], which relates the violation of the NEC to classical instabilities. Our metric convention is such that in a locally inertial frame $g_{\mu \nu}=\operatorname{diag}(1,-1, \ldots,-1)$.

\section{A. Scalar field model}

Consider the action

$$
S=\int d^{d} x|g|^{\frac{1}{2}}[Q(X)-V(\Phi)],
$$

where $X=\phi_{a, \mu} \phi^{a, \mu}$ and $\Phi=\phi_{a} \phi^{a}$. Throughout, commas indicate partial derivatives. By setting the variation of the action to zero, we find the equations of motion for the fields $\phi_{a}$ :

$$
\nabla_{\mu}\left(Q_{X} \phi^{a, \mu}\right)=-V_{\Phi} \phi^{a}
$$

In what follows, we assume that we have found a solution $\phi_{a}(x)$ to these equations of motion, and the quantities $Q_{X}$, etc. are evaluated at this solution. 


\section{Stability}

In order to determine whether the solution $\phi_{a}(x)$ to the equations of motion is stable against small fluctuations $\delta \phi_{a}$, we consider the second variation of the action,

$$
\delta^{2} S=\int d^{d} x|g|^{\frac{1}{2}}\left(\delta^{2} Q-\delta^{2} V\right)
$$

where

$$
\begin{aligned}
& \delta^{2} Q=\left(2 Q_{X} \delta^{a b} g^{\mu \nu}+4 Q_{X X} \phi^{a, \mu} \phi^{b, \nu}\right) \delta \phi_{a, \mu} \delta \phi_{b, \nu} \\
& \delta^{2} V=\left(2 V_{\Phi} \delta^{a b}+4 V_{\Phi \Phi} \phi^{a} \phi^{b}\right) \delta \phi_{a} \delta \phi_{b}
\end{aligned}
$$

We now use a locally inertial frame. For $\delta^{2} \mathcal{L}$, the canonical momentum is

$$
\delta \pi^{a}=4 Q_{X} \delta \phi^{a, 0}+8 Q_{X X} \phi^{a, 0} \phi^{b, \nu} \delta \phi_{b, \nu}
$$

and the Hamiltonian is $\delta^{2} \mathcal{H}=\delta^{2} K+\delta^{2} V$. Here the kinetic term is

$$
\begin{aligned}
\delta^{2} K & =\left(2 Q_{X} \delta^{a b}+4 Q_{X X} \phi^{a, 0} \phi^{b, 0}\right) \delta \phi_{a, 0} \delta \phi_{b, 0} \\
& +\left(2 Q_{X} \delta^{a b} \delta^{i j}-4 Q_{X X} \phi^{a, i} \phi^{b, j}\right) d \phi_{a, i} \delta \phi_{b, j},
\end{aligned}
$$

where $\delta \phi_{a, 0}$ are found by solving Eq. (6),

$$
\begin{aligned}
\delta \phi_{a, 0} & =\frac{1}{4 Q_{X}}\left(\delta^{b}{ }_{a}-\frac{4 Q_{X X} \phi^{b, 0} \phi_{a, 0}}{2 Q_{X}+4 Q_{X X} \phi_{c, 0} \phi^{c, 0}}\right) \\
& \times\left(\delta \pi_{b}-8 Q_{X X} \phi_{b, 0} \phi^{c, i} \delta \phi_{c, i}\right) .
\end{aligned}
$$

We are interested in finding a sufficient condition for the solutions of this model to be unstable. Moreover, we are free to investigate any set of variations we like. In particular, let us consider a set of variations such that $\delta \phi_{1, \mu} \neq 0$ and $\delta \phi_{a, \mu}=0(a \neq 1)$. In order to obtain a simple condition for instability, we further restrict $d-1$ quantities $\delta \phi_{1, i}$ to satisfy the following equation:

$$
\left(\phi^{1,0} \delta \phi_{1,0}\right)^{2}=\left(\phi^{1, i} \delta \phi_{1, i}\right)^{2} .
$$

For this choice of variations,

$$
\delta^{2} K=Q_{X}\left[\left(\delta \phi_{1,0}\right)^{2}+\sum_{i}\left(\delta \phi_{1, i}\right)^{2}\right] .
$$

We see that the classical solution $\phi_{a}(x)$ is unstable for $Q_{X}<0$. Specifically, it is unstable to the formation of gradients because the system can lower its kinetic energy by creating these gradients, while leaving the potential energy fixed. See Fig. 1 for a sketch.

\section{2. $N E C$}

Now let us consider the NEC for the model (10). The NEC states that $T_{\mu \nu} n^{\mu} n^{\nu} \geq 0$ for any null vector $n^{\mu}$. The energy-momentum tensor that couples to gravity is

$$
T_{\mu \nu}=-\mathcal{L} g_{\mu \nu}+2 \mathcal{L}_{g^{\mu \nu}} .
$$
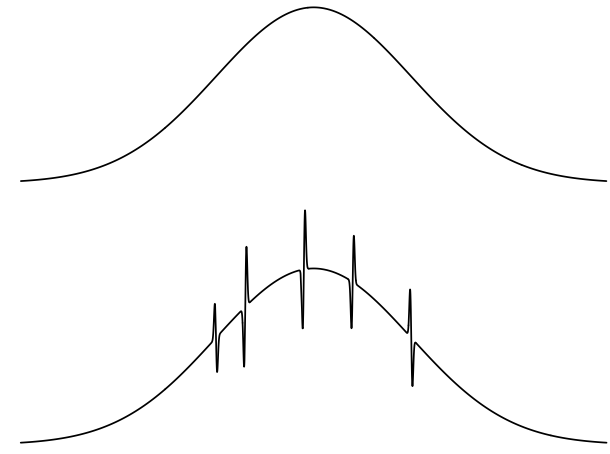

FIG. 1: In a model that violates the NEC, an initially smooth field configuration (top) is unstable. The system can lower its total energy by developing gradients (bottom), thereby decreasing its kinetic energy, while leaving its potential energy unchanged.

Computing the derivative $\mathcal{L}_{g^{\mu \nu}}=Q_{X} \phi^{a}{ }_{, \mu} \phi_{a, \nu}$, we see that the NEC requires

$$
Q_{X} \sum_{a}\left(n^{\mu} \phi_{a, \mu}\right)^{2} \geq 0
$$

and therefore,

$$
Q_{X} \geq 0 .
$$

Comparing Eqs. (10) and (13), we conclude that the violation of the NEC in this model implies that the classical solutions to the equations of motion are unstable to the formation of gradients.

\section{B. Gauge field model}

Consider the action

$$
S=\int d^{4} x|g|^{\frac{1}{2}}\left[Q(Y)+\theta|g|^{-\frac{1}{2}} \epsilon^{\mu \nu \rho \sigma} F_{\mu \nu} F_{\rho \sigma}\right],
$$

where $\theta$ is a dimensionless parameter, $|g|^{-\frac{1}{2}} \epsilon^{\mu \nu \rho \sigma}$ is the totally antisymmetric tensor in curved spacetime, $Y=$ $F_{\mu \nu} F^{\mu \nu}$, and $F_{\mu \nu}=A_{\nu ; \mu}-A_{\mu ; \nu}=A_{\nu, \mu}-A_{\mu, \nu}$. A semicolon indicates the covariant derivative appropriate for curved space, and the last equality holds because the connection has no torsion. Classical electrodynamics corresponds to $Q(Y)=-\frac{1}{4} Y$ and $\theta=0$.

As before, the equations of motion are found by setting the variation of the action to zero and integrating by parts. Here the equations of motion for $A_{\mu}$ are

$$
\nabla_{\mu}\left(Q_{Y} F^{\mu \nu}+\theta|g|^{-\frac{1}{2}} \epsilon^{\mu \nu \rho \sigma} F_{\rho \sigma}\right)=0 .
$$

In what follows we will assume that we have found a solution $A_{\mu}(x)$ to these equations of motion. 


\section{Stability}

As in the stability analysis for the scalar field model of the previous section, we consider the second variation of the action. Since the Lagrangian of (14) has no dependence on the gauge field $A_{\mu}$, this is simply

$$
\delta^{2} S=\int d^{d} x|g|^{\frac{1}{2}} \mathcal{L}_{A_{\alpha ; \mu} A_{\beta ; \nu}} \delta A_{\alpha ; \mu} \delta A_{\beta ; \nu}
$$

Derivatives of $\mathcal{L}$ with respect to the covariant derivative are equal to those with respect to the regular derivative, which in turn are proportional to derivatives with respect to the field strength, $\mathcal{L}_{A_{\alpha ; \mu} A_{\beta ; \nu}}=\mathcal{L}_{A_{\alpha, \mu} A_{\beta, \nu}}=4 \mathcal{L}_{F_{\alpha \mu} F_{\beta \nu}}$. Antisymmetry of $F_{\alpha \mu}$ is used to show the second equality. Therefore, to evaluate Eq. (16), we compute the derivatives of the Lagrangian with respect to the field strength. Again using the antisymmetry of $F_{\alpha \mu}$ :

$$
\mathcal{L}_{F_{\alpha \mu} F_{\beta \nu}}=M^{\alpha \beta} g^{\mu \nu}+N^{\alpha \mu \beta \nu},
$$

where we have defined

$$
\begin{aligned}
M^{\alpha \beta}= & 4 Q_{Y} g^{\alpha \beta} \\
N^{\alpha \mu \beta \nu}= & -4 Q_{Y} g^{\alpha \nu} g^{\beta \mu}+16 Q_{Y Y} F^{\alpha \mu} F^{\beta \nu} \\
& +8 \theta|g|^{-\frac{1}{2}} \epsilon^{\alpha \mu \beta \nu} .
\end{aligned}
$$

The second variation of the Lagrangian now becomes

$$
\delta^{2} \mathcal{L}=\left(M^{\alpha \beta} g^{\mu \nu}+N^{\alpha \mu \beta \nu}\right) \delta A_{\alpha ; \mu} \delta A_{\beta ; \nu}
$$

For the Lagrangian (20) the canonical momentum is

$$
\delta \pi^{\alpha}=2 M^{\alpha \beta} \delta A_{\beta ; 0}+2 N^{\alpha 0 \beta \nu} \delta A_{\beta ; \nu},
$$

where we use a locally inertial frame. This allows us to write

$$
\begin{aligned}
\delta^{2} \mathcal{H} & =\left(M^{\alpha \beta}+N^{\alpha 0 \beta 0}\right) \delta A_{\alpha ; 0} \delta A_{\beta ; 0} \\
& +\left(M^{\alpha \beta} \delta^{i j}-N^{\alpha i \beta j}\right) \delta A_{a ; i} \delta A_{\beta ; j}
\end{aligned}
$$

and $\delta A_{\alpha ; 0}$ are related to $\delta \pi_{\alpha}$ and $\delta A_{\alpha ; i}$ through Eq. (21). To show that this model can be unstable, we choose variations such that $\delta A_{1 ; \mu} \neq 0$ and $\delta A_{\alpha ; \mu}=0(\alpha \neq 1)$. We further restrict $d-1$ quantities $\delta A_{1 ; i}$ to satisfy the following equation:

$$
N^{1010} \delta A_{1 ; 0} \delta A_{1 ; 0}=N^{1 i 1 j} \delta A_{1 ; i} \delta A_{1 ; j}
$$

Therefore,

$$
\begin{aligned}
\delta^{2} \mathcal{H} & =M^{11} \delta A_{1 ; 0} \delta A_{1 ; 0}+M^{11} \delta^{i j} \delta A_{1 ; i} \delta A_{1 ; j} \\
& =-4 Q_{Y}\left[\left(\delta A_{1 ; 0}\right)^{2}+\sum_{i}\left(\delta A_{1 ; i}\right)^{2}\right]
\end{aligned}
$$

We see that if $Q_{Y}>0$ there is the same type of instability as in the scalar field case, independent of the value of $\theta$ in the $F \tilde{F}$ term. Note that in this model the total energy is simply the kinetic energy because there is no potential energy, i.e., no dependence on the field $A_{\mu}$.
2. $N E C$

In order to determine whether this model violates the NEC, we compute the following derivative

$$
\mathcal{L}_{g^{\mu \nu}}=\frac{1}{2} M^{\alpha \beta} F_{\alpha \mu} F_{\beta \nu}+\frac{1}{2} \theta g_{\mu \nu}|g|^{-\frac{1}{2}} \epsilon^{\alpha \beta \rho \sigma} F_{\alpha \beta} F_{\rho \sigma},
$$

where we have used $\partial|g| / \partial g^{\mu \nu}=-|g| g_{\mu \nu}$. From Eq. (25), we see that for this model the NEC requires

$$
Q_{Y} \Psi_{\alpha} \Psi^{\alpha} \geq 0
$$

where $\Psi_{\alpha}=n^{\mu} F_{\alpha \mu}$. Note that the $F \tilde{F}$ term does not enter into the NEC. We can determine the sign of $\Psi_{\alpha} \Psi^{\alpha}$ as follows. First notice

$$
n^{\alpha} \Psi_{\alpha}=n^{\alpha} n^{\mu} F_{\alpha \mu}=0
$$

by antisymmetry of $F_{\alpha \mu}$. Now use a locally inertial frame, and let $\Psi_{\alpha}=\left(\Psi_{0}, \Psi\right)$ and $n_{\alpha}=(|\mathbf{n}|, \mathbf{n})$. Then Eq. (27) implies

$$
n^{\alpha} \Psi_{\alpha}=|\mathbf{n}| \Psi_{0}-|\mathbf{n}||\Psi| \cos \varphi=0
$$

which means that $\Psi_{\alpha}$ is non-timelike, $\Psi_{\alpha} \Psi^{\alpha} \leq 0$. Therefore, Eq. (26) implies that the NEC is violated if $Q_{Y}>0$. As noted below Eq. (24), $Q_{Y}>0$ is also a sufficient condition for the model to be unstable. Thus, the violation of the NEC in this model implies that the classical solutions to the equations of motion are unstable to the formation of gradients.

\section{General case}

We now give an extended version of the general proof of the classical field theory result found in Ref. 1]. Consider a theory of scalar, $\phi_{a}$, and gauge, $A_{a \alpha}$, fields in a fixed $d$-dimensional spacetime with the metric $g_{\mu \nu}$. 1

\footnotetext{
${ }^{1}$ In our analysis we assume a fixed, but possibly non-trivial, spacetime metric $g_{\mu \nu}$. We study local instabilities of the matter fields propagating on that spacetime background that would manifest themselves on microphysical timescales. It is always possible to work in an inertial frame at any particular point in spacetime. One might wonder whether backreaction of the metric might dampen the growth of an instability. Since the principle of equivalence (which guarantees the existence of an inertial frame) applies everywhere, it is easy to see that stability cannot be restored unless the null energy condition ceases to be violated. That is, although backreaction could in principle decrease the magnitude of the negative eigenvalue governing the instability, the eigenvalue cannot go to zero or change sign unless the null energy condition is no longer violated. Furthermore, the amount by which backreaction might decrease the growth of the instability is suppressed by the Planck scale (this is easy to deduce from the Einstein equations), so it is negligible except in circumstances in which quantum gravitational effects are important, and known methods cease to apply.
} 
We limit ourselves to theories whose equations of motion are second order differential equations, so the Lagrangian density $\mathcal{L}$ for the system is assumed to depend only on the the scalar fields $\phi_{a}$, their covariant derivatives $D_{\mu} \phi_{a}$, and the gauge field strengths $F_{a \mu \nu}$. The scalars may transform in any representation of the gauge group. We impose Lorentz invariance on $\mathcal{L}$, but do not require overall gauge invariance. That is, we allow for fixed tensors with gauge indices (but no Lorentz indices) which can be contracted with the fields, as is the case, e.g., in models with spontaneous symmetry breaking.

Although we consider minimal coupling between matter fields and gravity, non-minimal models of the type below are related to minimal models by conformal transformation [8]. Indeed, consider the non-minimally coupled action

$$
S=\int d^{d} x|g|^{\frac{1}{2}}\left[\frac{1}{2} B\left(\phi_{a}\right) R+\mathcal{L}\left(\phi_{a}, D_{\mu} \phi_{a}, F_{a \mu \nu}\right)\right],
$$

where $R$ is the scalar curvature. For a single minimally and non-minimally coupled scalar, $B=1$ and $B=1-\frac{1}{2} \xi \phi^{2}$, respectively. Under a conformal transformation $g_{\mu \nu} \rightarrow \tilde{g}_{\mu \nu}=\Omega^{2} g_{\mu \nu}$, the action (29) transforms as follows:

$$
\begin{aligned}
S \rightarrow \tilde{S} & =\int d^{d} x|g|^{\frac{1}{2}} \Omega^{d}\left\{\tilde{\mathcal{L}}+\frac{1}{2} B \Omega^{-2}[R\right. \\
& \left.\left.-2(d-1) \square \ln \Omega-(d-2)(d-1)(\nabla \ln \Omega)^{2}\right]\right\}
\end{aligned}
$$

where $\tilde{\mathcal{L}}$ is within the same class as $\mathcal{L}$, and all indices are raised and lowered with the untransformed metric. Notice that if we choose $\Omega=B^{1 /(2-d)}$ and integrate Eq. (30) by parts, the conformally transformed action becomes the action for minimally coupled scalars with a Lagrangian density of no larger generality than $\mathcal{L}$. (Derivatives of $B$ give terms involving gradients of the scalar fields; after adding these with additional multiplication by $\phi_{a}$-dependent terms, the resulting Lagrangian is still within a class of $\mathcal{L}$ considered here.) The conformally transformed action is

$$
\tilde{S}=\int d^{d} x|g|^{\frac{1}{2}}\left[\frac{1}{2} R+B^{\frac{d}{2-d}} \tilde{\mathcal{L}}-\frac{d-1}{2(d-2)}(\nabla \ln B)^{2}\right]
$$

Thus, there is no need to consider non-minimally coupled models of Eq. (29) separately.

For the action

$$
S=\int d^{d} x|g|^{\frac{1}{2}} \mathcal{L}\left(\phi_{a}, D_{\mu} \phi_{a}, F_{a \mu \nu}\right)
$$

to be stationary, its first variation has to vanish, $\delta S=0$. This leads to the equations of motion for the fields $\phi_{a}$ and $A_{a \alpha}$; in the classical analysis we assume that we have found solutions to these equations, about which we expand. See Fig. 2 for a flow chart of the method that we use to show a connection between violation of the NEC and instability.

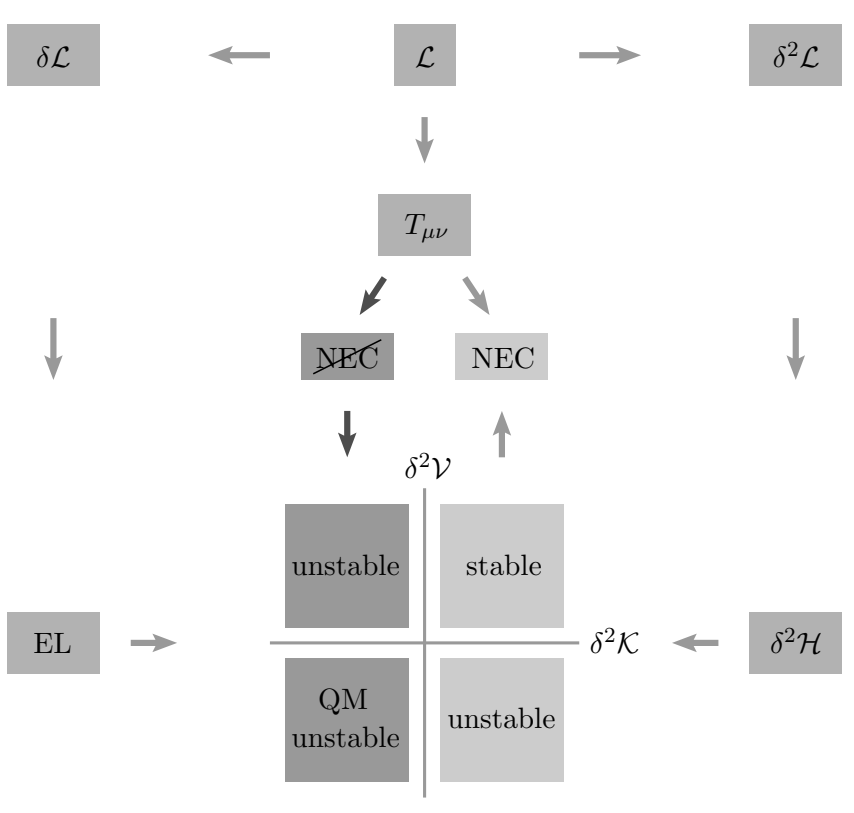

FIG. 2: A flow chart of the general version of the proof that for models described by the action (32), only solutions satisfying the NEC can be stable.

\section{NEC}

We use the notation $\psi_{A \mu}=\left(D_{\mu} \phi_{a}, F_{a \alpha \mu}\right)$, where the abstract index $A$ runs over both Lorentz and color indices, as well as type of field. For example, in Sec. ПA the index $A$ is a color index, $\psi_{A \mu}=\phi_{a, \mu}$, while in Sec. IIB it is a Lorentz index, $\psi_{A \mu}=F_{\alpha \mu}$. In general, for a model with $N_{\mathrm{s}}$ scalar fields and $N_{\mathrm{g}}$ gauge fields in $d$ dimensions, $A$ runs from 1 to $N_{\mathrm{s}}+\frac{1}{2}(d-1) N_{\mathrm{g}}$.

Assuming that $\psi_{A \mu}$ and $g^{\mu \nu}$ are independent variables, we now prove that there is a relation between the derivatives of $\mathcal{L}$ with respect to them. Since we consider only Lorentz invariant models, the Lagrangian can be written in terms of a number of Lorentz invariant quantities:

$$
\mathcal{L}=\mathcal{L}\left(X_{1}, X_{2}, \ldots\right)
$$

Objects represented in Fig. 3] are the simplest examples of $X_{n}$. Each dot represents a Lorentz index, and a line connecting them denotes contraction using the metric. Rectangles (with two indices) are field strengths, small circles covariant derivatives of scalar fields, and large circles epsilon tensors. All Lorentz indices are ultimately contracted, and we suppress color indices for simplicity. As we will show, the relation between the derivatives of 


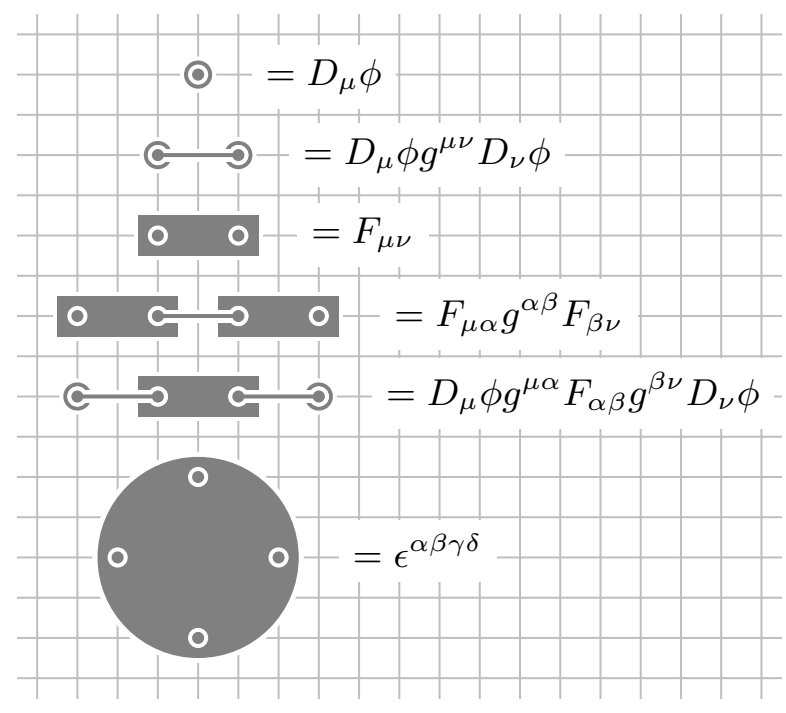

FIG. 3: Representation of simple Lorentz invariants that the Lagrangian may depend upon. Each dot represents a Lorentz index, and a line connecting them denotes contraction using the metric. Rectangles (with two indices) are field strengths, small circles covariant derivatives of scalar fields, and large circles epsilon tensors. All Lorentz indices are ultimately contracted, and we suppress color indices for simplicity.

$\mathcal{L}$ is

$$
\begin{aligned}
\mathcal{L}_{g^{\mu \nu}} & =\sum_{n} \mathcal{L}_{X_{n}}\left(X_{n}\right)_{g^{\mu \nu}} \\
& =\frac{1}{2} \sum_{n}\left(M_{(n)}{ }^{A B} \psi_{A \mu} \psi_{B \nu}+g_{\mu \nu} K_{(n)}\right) \\
& =\frac{1}{2} M^{A B} \psi_{A \mu} \psi_{B \nu}+\frac{1}{2} g_{\mu \nu} K, \\
\mathcal{L}_{\psi_{A \mu}} & =\sum_{n} \mathcal{L}_{X_{n}}\left(X_{n}\right)_{\psi_{A \mu}} \\
& =\sum_{n}\left(M_{(n)}{ }^{A B} \psi_{B}{ }^{\mu}+\epsilon^{\mu \nu_{2} \cdots \nu_{d}} L_{(n)}{ }^{A}{ }_{\nu_{2} \cdots \nu_{d}}\right) \\
& =M^{A B}{\psi_{B}}^{\mu}+\epsilon^{\mu \nu_{2} \cdots \nu_{d}} L_{\nu_{2} \cdots \nu_{d}}^{A},
\end{aligned}
$$

where quantities $M_{(n)}, K_{(n)}$ and $L_{(n)}$ originate from the dependence of $\mathcal{L}$ on $X_{n}$. The first term in Eq. (34) is obtained by noting that for each and every $g^{\mu \nu}$ in $\mathcal{L}$ there are two $\psi_{\text {s }}$ attached to it. The second term is present if $\mathcal{L}$ has a term involving the curved space totally antisymmetric tensor $|g|^{-\frac{1}{2}} \epsilon^{\nu_{1} \cdots \nu_{d}}$, again recalling that the derivative of the determinant of the metric is proportional to the metric. Similarly, differentiation with respect to $\psi_{A \mu}$ yields the $M$ and $L$ terms in Eq. (35).

To prove this relation for the most general case, we consider the most general Lagrangian of the type considered in this paper, represented by Fig. (4 The notation is the same as in Fig. 3 with the addition that $Z$ denotes the elements of the Lagrangian which are shaded gray. We focus our attention on the term represented by the elements which are shaded black.

Indeed, consider the portion of Fig. [4 whose elements

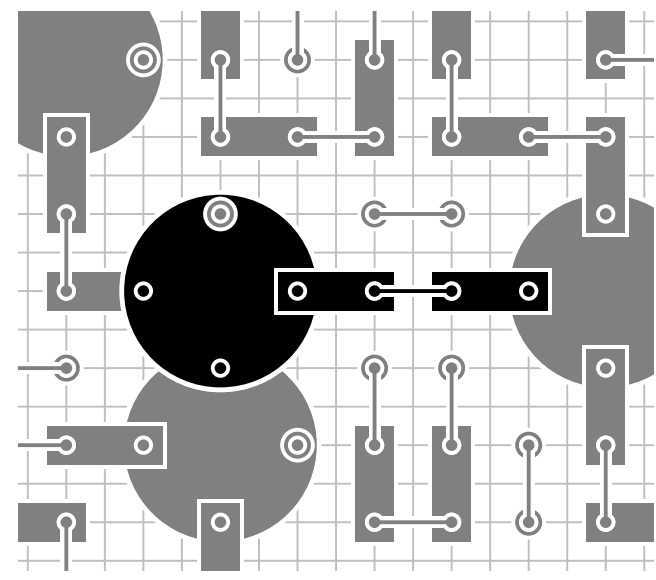

FIG. 4: Representation of the most general Lagrangian of the type considered in this paper. The notation is the same as in Fig. 3. We focus on the elements shaded black. In graphical terms, $M$ for this term in the Lagrangian can be obtained from the figure by simply removing these elements. $Z$ represents the remainder of the Lagrangian.

are shaded black; it equals

$$
\mathcal{L}=|g|^{-\frac{1}{2}} \epsilon^{\alpha \cdots} F_{\alpha \mu} g^{\mu \nu} F_{\nu \beta} g^{\beta \gamma} Z_{\gamma \cdots}
$$

(Again, we suppress color indices for simplicity, as they do not affect the proof.) By differentiation, we obtain Eqs. (34) and (35) with

$$
\begin{aligned}
M^{\alpha \beta} & =-|g|^{-\frac{1}{2}}\left(\epsilon^{\alpha \cdots} g^{\beta \gamma}+\epsilon^{\beta \cdots} g^{\alpha \gamma}\right) Z_{\gamma \cdots}, \\
K & =|g|^{-\frac{1}{2}} \epsilon^{\alpha \cdots} F_{\alpha \rho} g^{\rho \sigma} F_{\sigma \beta} g^{\beta \gamma} Z_{\gamma \cdots}, \\
L^{\alpha} \ldots & =-|g|^{-\frac{1}{2}} g^{\alpha \rho} F_{\rho \beta} g^{\beta \gamma} Z_{\gamma \cdots}
\end{aligned}
$$

Note the derivative $\mathcal{L}_{F_{\nu \beta}}$ generates a contribution to $M$ which is matched by a corresponding contribution from $\mathcal{L}_{g^{\beta \gamma}}$. Other contractions of fields with $g_{\mu \nu}$ (i.e., as indicated in the figure) can be analyzed similarly. In graphical terms, $M$ can be obtained from the figure by simply removing the elements shaded black.

From Eq. (34), the NEC requires

$$
\Psi_{A} M^{A B} \Psi_{B} \geq 0
$$

where $\Psi_{A}=\psi_{A \mu} n^{\mu}$. Thus, to satisfy the NEC, $M^{A B}$ has to be positive semidefinite on the subspace spanned by $\Psi_{A}$. This property is crucial for stability of solutions, to which we now turn.

\section{Stability}

To study the stability of the solution $\psi_{A}(x)$, we consider the second variation of the action,

$$
\begin{array}{r}
\delta^{2} S=\int d^{d} x|g|^{\frac{1}{2}}\left(\mathcal{L}_{\psi_{A} \psi_{B}} \delta \psi_{A} \delta \psi_{B}\right. \\
\left.+2 \mathcal{L}_{\psi_{A} \psi_{B ; \lambda}} \delta \psi_{A} \delta \psi_{B ; \lambda}+\mathcal{L}_{\psi_{A ; \mu} \psi_{B ; \nu}} \delta \psi_{A ; \mu} \delta \psi_{B ; \nu}\right) .
\end{array}
$$


Here quantities $\mathcal{L}_{\psi_{A}}=\partial \mathcal{L} / \partial \psi_{A}$, etc. are evaluated at $\psi_{A}(x)$. Also notice that $\psi_{A ; \mu}=\left(D_{\mu} \phi_{a}, A_{a \alpha ; \mu}\right)$, the covariant derivatives of $\psi_{A}$, are different from $\psi_{A \mu}=$ $\left(D_{\mu} \phi_{a}, F_{a \alpha \mu}\right)$.

Differentiating Eq. (35) we find

$$
\mathcal{L}_{\psi_{A \mu} \psi_{B \nu}}=M^{A B} g^{\mu \nu}+N^{A \mu B \nu} .
$$

The separation of the second derivative into the first and second terms in Eq. (42) is natural: $g^{\mu \nu}$ appears only in the first term, and $N$ represents all remaining terms. $N$ contains terms obtained by differentiating $M$ and $L$ with respect to $\psi_{B \nu}$, plus additional terms if $\psi$ is a field strength, as in Eq. (19). The $\nu$ index obtained from these $\psi_{B \nu}$ derivatives is attached to a field and not the metric $g^{\mu \nu}$. ( $L$ does not contain an epsilon tensor since $Z$ does not.)

Next notice that even though $\psi_{A \mu}$ and $\psi_{A ; \mu}$ differ, the derivatives of $\mathcal{L}$ with respect to them are related due to the form of the action (32). Thus, the second variation of the Lagrangian in Eq. (41) becomes

$$
\begin{aligned}
\delta^{2} \mathcal{L} & =\left(M^{A B} g^{\mu \nu}+N^{A \mu B \nu}\right) \delta \psi_{A ; \mu} \delta \psi_{B ; \nu} \\
& +2 \mathcal{L}_{\psi_{A} \psi_{B ; \lambda}} \delta \psi_{A} \delta \psi_{B ; \lambda}-\delta^{2} V
\end{aligned}
$$

where $\delta^{2} V=-\mathcal{L}_{\psi_{A} \psi_{B}} \delta \psi_{A} \delta \psi_{B}$.

Let us use a locally inertial frame. For the Lagrangian (43), the canonical momentum is

$$
\begin{aligned}
\delta \pi^{A}=2 M^{A B} \delta \psi_{B ; 0} & +2 N^{A 0 B \nu} \delta \psi_{B ; \nu} \\
& +2 \mathcal{L}_{\psi_{B} \psi_{A ; 0}} \delta \psi_{B},
\end{aligned}
$$

which leads to the following effective Hamiltonian for fluctuations about the classical solution:

$$
\delta^{2} \mathcal{H}=\delta^{2} K+\delta^{2} V-2 \mathcal{L}_{\psi_{A} \psi_{B ; i}} \delta \psi_{A} \delta \psi_{B ; i},
$$

where the kinetic term is

$$
\begin{aligned}
\delta^{2} K & =\left(M^{A B}+N^{A 0 B 0}\right) \delta \psi_{A ; 0} \delta \psi_{B ; 0} \\
& +\left(M^{A B} \delta^{i j}-N^{A i B j}\right) \delta \psi_{A ; i} \delta \psi_{B ; j} .
\end{aligned}
$$

Here $\delta \psi_{A ; 0}$ are functions of $\delta \pi_{B}, \delta \psi_{B}$, and $\delta \psi_{B ; i}$ as found from Eq. (44).

We now prove that nonnegativeness of the kinetic term $\delta^{2} K$ implies positive semidefiniteness of the matrix $M^{A B}$. Indeed, suppose that $M^{A B}$ is not positive semidefinite. In such a case, the matrix $M^{A B}$ has at least one negative eigenvalue, which means that there is a basis in which the matrix is diagonal with at least one negative entry, $M^{A B}=\operatorname{diag}\left(m_{1}, \ldots, m_{n}\right)\left(m_{1}<0\right)$. Let us choose field variations which are nonzero only in the direction of the negative eigenvalue: $\delta \psi_{1 ; \mu} \neq 0$ and $\delta \psi_{A ; \mu}=0(A>1)$. We further restrict $d-1$ quantities $\delta \psi_{1 ; i}$ to satisfy the following equation:

$$
N^{1010} \delta \psi_{1 ; 0} \delta \psi_{1 ; 0}=N^{1 i 1 j} \delta \psi_{1 ; i} \delta \psi_{1 ; j}
$$

These conditions make the kinetic term in Eq. 45) negative,

$$
\delta^{2} K=m_{1}\left[\left(\delta \psi_{1 ; 0}\right)^{2}+\sum_{i}\left(\delta \psi_{1 ; i}\right)^{2}\right]<0,
$$

thus proving that in order for $\delta^{2} K$ to be nonnegative, the matrix $M^{A B}$ has to be positive semidefinite.

Note that if the background field leaves an unbroken rotational symmetry - i.e., there are $d-2$ or fewer independent vacuum expectation values $\psi_{\mu}$-one can find a solution to Eq. (47) in any frame, by taking $\delta \psi_{1 ; 0}=0$ and the spacelike fluctuations to have support only in the direction(s) orthogonal to all of the $\psi_{\mu}$. In the remaining cases (which, in particular, have no unbroken rotational symmetries) there may be Lorentz frames in which no solution to Eq. (47) exists. The examples given by Ref. 6 are in this category. In Refs. [6] and [7], it is emphasized that superluminal modes play a crucial role in permitting stability and NEC violation simultaneously. However, models with superluminal modes are not causal. In the appendix, we show that the assumption of causality is strong enough to restore the direct connection between NEC violation and instability.

If the kinetic energy is negative, then the system described by the Hamiltonian (45) is (locally) unstable. The linear term in Eq. 45) can never act to stabilize the system. Therefore, in order to determine whether the system is unstable, we focus only on the quadratic terms. If the potential energy is positive then small perturbations will cause the classical solutions to grow exponentially away from the original stationary point. However, it is possible to have classical stability if one chooses the potential energy to be negative; in this case we have an upside-down potential with negative kinetic term, or a "phantom". Such models necessarily exhibit quantum instabilities 11]. Using the result established in the previous paragraph, we thus conclude that solutions to the theory given by the action (32) are stable only if the matrix $M^{A B}$ is positive semidefinite.

Combining the relations between nonnegativeness of $\delta^{2} K$ and positive semidefiniteness of $M^{A B}$ on one hand, and the NEC and positive semidefiniteness of $M^{A B}$ on the the other hand, we conclude that for the theory given by the action (32), only solutions satisfying the NEC can be stable.

\section{QUANTUM FIELD THEORIES}

We can deduce similar results for quantum systems. Suppose there exists a quantum state $|\alpha\rangle$ and a null vector $n^{\mu}$ such that the NEC is violated in a quantum averaged sense:

$$
\left\langle\alpha\left|T_{\mu \nu}\right| \alpha\right\rangle n^{\mu} n^{\nu}=\left\langle\alpha\left|M^{A B} \Psi_{A} \Psi_{B}\right| \alpha\right\rangle<0 .
$$

Define a basis $|\phi\rangle$ in which the operator $\mathcal{M}=M^{A B} \Psi_{A} \Psi_{B}$ is diagonal:

$$
\mathcal{M}|\phi\rangle=m(\phi)|\phi\rangle .
$$


By orthonormality of $|\phi\rangle$, we see that violation of the quantum averaged NEC implies

$$
\begin{aligned}
\langle\alpha|\mathcal{M}| \alpha\rangle & =\sum_{\phi \phi^{\prime}}\langle\alpha \mid \phi\rangle\left\langle\phi|\mathcal{M}| \phi^{\prime}\right\rangle\left\langle\phi^{\prime} \mid \alpha\right\rangle \\
& =\sum_{\phi}|\langle\alpha \mid \phi\rangle|^{2} m(\phi)<0 .
\end{aligned}
$$

This means that there exist eigenstates $|\phi\rangle$, whose overlap with $|\alpha\rangle$ is non-zero and on which the operator $\mathcal{M}$ has negative eigenvalues. This requires that $M^{A B}$ and hence $\delta^{2} K$ is not positive semidefinite; by continuity, this must also be the case in a ball $B$ in the Hilbert space of $|\phi\rangle$.

As a further consequence, we can conclude that a state $|\alpha\rangle$ in which the quantum averaged NEC is violated cannot be the ground state [12]. Suppose that $|\alpha\rangle$ is an energy eigenstate: $H|\alpha\rangle=E_{\alpha}|\alpha\rangle$. An elementary result from quantum mechanics is that $|\alpha\rangle$ can be the ground state only if

$$
E_{\alpha}=\langle\alpha|H| \alpha\rangle \leq\left\langle\alpha^{\prime}|H| \alpha^{\prime}\right\rangle
$$

for all normalized states $\left|\alpha^{\prime}\right\rangle$ which need not be energy eigenstates. However, it is possible to reduce the expectation value of $H$ by perturbing $|\alpha\rangle$. Specifically, we adjust $|\alpha\rangle$ only in the ball $B$, where we know from Eqs. (46)- (48) that there are perturbations which reduce the expectation of the kinetic energy without changing the expectation of the potential. For a pictorial representation of this, see Fig. 11 where now the two different graphs are sketches of two eigenstates of the operator $\mathcal{M}$. Let the top one be $\left|\phi_{1}\right\rangle$ and the bottom $\left|\phi_{2}\right\rangle$. Expand the state $|\alpha\rangle$ in the basis of these eigenstates:

$$
|\alpha\rangle=\sum_{i} c_{i}\left|\phi_{i}\right\rangle
$$

Since by assumption the state $|\alpha\rangle$ violates the quantum averaged NEC, the magnitude of coefficients of smooth eigenstates, e.g. $\left|\phi_{1}\right\rangle$, will decrease, while those of eigenstates with large gradients, e.g. $\left|\phi_{2}\right\rangle$, will increase, until the quantum averaged NEC is no longer violated. Thus, a state $|\alpha\rangle$ which violates the quantum averaged NEC cannot be the ground state because perturbations within the ball $B$ can lower the expectation value of the energy.

Note that the discussion above is in terms of unrenormalized (bare) quantities. The renormalized expectation

$$
\left\langle\alpha\left|\mathcal{M}_{\text {ren }}\right| \alpha\right\rangle=\langle\alpha|\mathcal{M}| \alpha\rangle-\langle 0|\mathcal{M}| 0\rangle,
$$

where $|0\rangle$ is the flat space QFT ground state, could be negative (e.g., as in the Casimir effect [2]), but this is possible only if $|\alpha\rangle$ is not the ground state.

In known cases of NEC violation, such as the Casimir vacuum or black hole spacetime, it is only the renormalized energy-momentum tensor which violates the NEC. As a simple example, consider a real scalar field $\phi$. The energy-momentum tensor is simply $T_{\mu \nu}=\partial_{\mu} \phi \partial_{\nu} \phi$ plus terms proportional to $g_{\mu \nu}$ which do not play a role in the
NEC. Then $\mathcal{M}=\left(n^{\mu} \partial_{\mu} \phi\right)^{2}$ is a Hermitian operator with positive eigenvalues. Therefore, its expectation value in any state is positive:

$$
\langle\alpha|\mathcal{M}| \alpha\rangle>0,
$$

for any $|\alpha\rangle$, including the Hartle-Hawking, Casimir or flat-space vacuum. We can verify this by direct calculation in $d=4$ dimensions, computing the energymomentum tensor using point-splitting regularization:

$$
\left\langle 0\left|\phi(x) \phi\left(x^{\prime}\right)\right| 0\right\rangle=-\frac{1}{4 \pi^{2}\left|x-x^{\prime}\right|^{2}},
$$

which yields

$$
\left\langle 0\left|T_{\mu \nu}\left(x, x^{\prime}\right)\right| 0\right\rangle n^{\mu} n^{\nu}=\frac{2\left[n^{\mu}\left(x-x^{\prime}\right)_{\mu}\right]^{2}}{\pi^{2}\left|x-x^{\prime}\right|^{6}} .
$$

So $\langle 0|\mathcal{M}| 0\rangle$ is manifestly positive. Note that this result holds even if the expectation is taken in an arbitrary state rather than $|0\rangle$, since the bare expectation is always dominated by the UV contribution. Now, had we taken a negative kinetic energy term for the scalar, the overall sign of $\mathcal{M}$ changes, allowing violation of the NEC. But, this model is clearly unstable, in accordance with our results.

Our results are summarized as follows, where $|\alpha\rangle$ is any quantum state, and only models of the type described by action (32) are considered: (1) $\langle\alpha|\mathcal{M}| \alpha\rangle<0$, i.e., the bare NEC is violated, implies that $|\alpha\rangle$ is locally unstable, (2) $\left\langle\alpha\left|\mathcal{M}_{\text {ren }}\right| \alpha\right\rangle<0$, i.e., the renormalized NEC is violated, implies that $|\alpha\rangle$ cannot be the global ground state.

\section{MODELS WITH FERMIONS}

In this section we present a generalized version of the treatment of fermions in [1]. Both Dirac and Weyl fermions with standard couplings to the bosonic fields of Sec. III may be included in the model without any effect on the results of that section. In the purely classical analysis, fermions play no role, while in the quantum case we can integrate them out to obtain their contribution to the energy-momentum tensor. We will see that this fermionic contribution always satisfies the NEC.

In addition to the bosonic part in the Lagrangian, we include the following fermionic part:

$$
\mathcal{L}^{(\mathrm{f})}=\bar{\psi}\left[i \not D-m\left(\phi_{a}\right)\right] \psi .
$$

A Dirac fermion $\psi$ couples to scalar and vector bosons via a covariant derivative $D_{\mu}=\nabla_{\mu}+A_{\mu}$ and an arbitrary real scalar function $m\left(\phi_{a}\right)$. We use Euclidean quantities obtained via substitutions $x^{0} \rightarrow-i x^{0}, A_{0} \rightarrow i A_{0}, \gamma^{0} \rightarrow$ $-i \gamma^{0}$, and find the effective Euclidean action $\Gamma$ from

$$
\begin{aligned}
e^{-\Gamma} & =\int \mathcal{D} \bar{\psi} \mathcal{D} \psi \exp \left[-\int d^{d} x|g|^{\frac{1}{2}} \mathcal{L}^{(\mathrm{f})}\right] \\
& =\operatorname{det}(i \not D-m) .
\end{aligned}
$$


The Euclidean operator $\not D$ is self-adjoint.

The imaginary part of the action vanishes since it is an odd function of $D$ and the trace of odd number of gamma matrices vanishes:

$$
\begin{aligned}
\operatorname{Im} \Gamma & =-\frac{1}{2} \operatorname{tr} \ln \left[(i \not D-m)(-i \not D-m)^{-1}\right] \\
& =i \operatorname{tr} \arctan \left(\not D m^{-1}\right)=0 .
\end{aligned}
$$

Using eigenvectors $\psi_{k}$ and eigenvalues $\lambda_{k}$ of the operator $i \not D-m$, the real part of the action becomes

$$
\begin{aligned}
\operatorname{Re} \Gamma & =-\frac{1}{2} \operatorname{tr} \ln [(-i \not D-m)(i \not D-m)] \\
& =-\frac{1}{2} \sum_{k} \ln \left|\lambda_{k}\right|^{2} .
\end{aligned}
$$

Since the operator $i \not D-m$ is not self-adjoint, the eigenvalues are complex and the eigenfunctions are not orthogonal. However, they can be normalized, $\int d^{d} x|g|^{\frac{1}{2}} \psi_{k}^{\dagger} \psi_{k}=$ 1. Using this normalization we have

$$
\left|\lambda_{k}\right|^{2}=\int d^{d} x|g|^{\frac{1}{2}} \psi_{k}^{\dagger}(-i \not D-m)(i \not D-m) \psi_{k} .
$$

For the purposes of calculating the contribution of $\operatorname{Re} \Gamma$ to the energy momentum tensor, it is enough to consider only those terms in Eq. (62) which contain $g^{\mu \nu}$ :

$$
\left|\lambda_{k}\right|^{2}=-\int d^{d} x|g|^{\frac{1}{2}} g^{\mu \nu}\left(D_{\mu} \psi_{k}\right)^{\dagger}\left(D_{\nu} \psi_{k}\right)+\cdots .
$$

We have used here anti-hermiticity of $D_{\mu}$. Thus, using the definition of the energy momentum tensor

$$
T_{\mu \nu}^{(\mathrm{f})}=2|g|^{-\frac{1}{2}} \frac{\delta \Gamma}{\delta g^{\mu \nu}},
$$

the fermionic contribution to the NEC expression is nonnegative:

$$
T_{\mu \nu}^{(\mathrm{f})} n^{\mu} n^{\nu}=\sum_{k}\left|\lambda_{k}\right|^{-2}\left(n^{\mu} D_{\mu} \psi_{k}\right)^{\dagger}\left(n^{\nu} D_{\nu} \psi_{k}\right) \geq 0 .
$$

The Weyl operator does not define an eigenvalue problem, as it maps a spinor into the opposite chirality; see e.g. [13]. However, the Weyl determinant can be defined as the square root of the corresponding Dirac determinant obtained by considering the Weyl fermion and a partner in the complex conjugate representation. The real part of the contribution to the energy-momentum tensor is exactly one half of the Dirac contribution considered previously. The presence of either type of fermion does not lead to violation of the NEC, and so the results of Sec. III hold in models with arbitrary fermionic and bosonic particle content.

\section{FLUIDS}

\section{A. Perfect Fluids}

A macroscopic system may be approximately described as a perfect fluid if the mean free path of its components is small compared to the length scale of interest. For the dark energy, this length scale is of cosmological size. A perfect fluid is described by the energy-momentum tensor

$$
T_{\mu \nu}=(\rho+p) u_{\mu} u_{\nu}-p g_{\mu \nu},
$$

where $\rho$ and $p$ are the energy density and pressure of the fluid in its rest frame, and $u_{\mu}$ is its velocity. Let $j^{\mu}=J u^{\mu}$ be the conserved current vector $\left(j_{; \mu}^{\mu}=0\right)$, and $J=\left(j_{\mu} j^{\mu}\right)^{\frac{1}{2}}$ the particle density.

The energy-momentum can be written [3, 14] as

$$
T_{\mu \nu}=\left(f-J f^{\prime}\right) g_{\mu \nu}+\left(f^{\prime} / J\right) j_{\mu} j_{\nu},
$$

where, comparing with Eq. (66), we have $\rho=f(J)$ and $p=J f^{\prime}-f$. The function $f(J)$ implicitly determines the equation of state. Also, prime indicates derivative with respect to $J$ at fixed temperature and volume.

The NEC for the tensor (67) becomes

$$
T_{\mu \nu} n^{\mu} n^{\nu}=\left(f^{\prime} / J\right)\left(j_{\mu} n^{\mu}\right)^{2} \geq 0 .
$$

Thus, perfect fluids with negative $f^{\prime}(J)$ violate the NEC. Below, we demonstrate that $f^{\prime}(J)<0$ implies an instability.

Recall that the speed of sound in a fluid is given by $c_{\mathrm{s}}=(d p / d \rho)^{\frac{1}{2}}=\left(J f^{\prime \prime} / f^{\prime}\right)^{\frac{1}{2}}$, and that complex $c_{\mathrm{s}}$ (or negative compressibility) implies a mechanical instability [15]. This instability is avoided only if $f^{\prime \prime}<0$. However, if $f^{\prime}$ and $f^{\prime \prime}$ are negative, then the fluid is unstable with respect to clumping. To see this, we first recall that the Helmholtz free energy $F$ is a Legendre transform of the internal energy $E$, i.e., $F=E-T S$, where $S$ is the entropy. In the thermodynamic limit, entropy scales with particle number $N=J V$, and so

$$
F=V f-J V T s
$$

where from above, $f(J)=\rho$ is the energy density, and $s=S / N$ is the specific entropy, which is a function of temperature but not particle number. Therefore,

$$
\left.\frac{\partial^{2} F}{\partial J^{2}}\right|_{T, V}=V f^{\prime \prime}
$$

Now consider two adjacent regions of the fluid with identical volumes. Suppose we transfer a small amount of matter $\delta J$ from one volume to another. Expand the change in free energy to second order in the number density:

$$
\begin{aligned}
\delta F_{1}+\delta F_{2} & =\frac{\partial F}{\partial J_{1}} \delta J_{1}+\frac{1}{2} \frac{\partial^{2} F}{\partial J_{1}^{2}}\left(\delta J_{1}\right)^{2} \\
& +\frac{\partial F}{\partial J_{2}} \delta J_{2}+\frac{1}{2} \frac{\partial^{2} F}{\partial J_{2}^{2}}\left(\delta J_{2}\right)^{2}+\cdots
\end{aligned}
$$

The linear terms cancel due to particle number conservation, $\delta J_{1}=-\delta J_{2}=\delta J$. Therefore, using Eq. (70), the resulting change in total free energy is given by

$$
\delta F=\frac{1}{2} V f^{\prime \prime}(J)(\delta J)^{2}<0 .
$$


We see that the system can decrease its free energy by clumping into over- and under-dense regions. This itself is an instability, which results in a runaway to infinitely negative free energy unless the assumption of negative $f^{\prime}$ or negative $f^{\prime \prime}$ ceases to hold. NEC violation either leads complex speed of sound or clumping instability.

\section{B. Beyond Fluids}

We can generalize our analysis beyond fluids, relaxing the assumption of the existence of a conserved current $j^{\mu}$, and requiring only energy conservation and statistical equilibrium.

Consider an isolated system at fixed energy $E$. If the system is ergodic it can (after a sufficiently long time) be described by a microcanonical ensemble, which uniformly samples phase space. If it has many degrees of freedom, the microcanonical ensemble is well approximated by a canonical ensemble with a Boltzmann weighting, in which the energy is allowed to fluctuate. In this case the thermodynamical relation $E=T S-p V$ is satisfied, with $T=\left.(\partial E / \partial S)\right|_{V}$. Dividing by the volume, we obtain $\rho+p=T \sigma$, where $\sigma=S / V$ is the positive-definite entropy density. Thus, for a system which is homogeneous and isotropic, i.e., one with energy-momentum tensor as in Eq. (66), violation of the NEC requires negative temperature, or entropy (number of states) which decreases with energy. For a field theory, this suggests either a negative kinetic energy term or an "upside-down" potential; if the kinetic and potential energies have opposite sign (the usual case), the number of states available to the system increases as energy is added, and the temperature is positive. Moreover, in order for the partition function (trace over Boltzmann weights) to converge, the kinetic energy term must be negative if the temperature is. This is because at any fixed potential energy there is an infinite volume of phase space with arbitrarily large gradients, i.e., kinetic energy. Therefore, if the kinetic energy term and the temperature are not of the same sign, the partition function does not converge.

An example of a condensed matter system with a negative temperature is a ferromagnet in an external magnetic field [16]. Beyond a certain threshold energy, the number of states available to the system decreases as energy is added, i.e., the temperature is negative. Due to the presence of an external magnetic field, however, the system is anisotropic; its energy-momentum tensor is not of the form of Eq. (66), and there is no connection between negative temperature and violation of the NEC. It is only for homogeneous, isotropic systems, e.g., systems of interest in cosmology, that this relation exists.

\section{CONCLUSION}

We have exhibited a direct connection between violation of the NEC and instability in a wide variety of mod- els. One important application is to the dark energy, whose equation of state $w$ should be no more negative than -1 in order not to violate the NEC.

In classical field theory-including all Lorentzinvariant models involving both minimally and nonminimally coupled scalar and gauge fields with secondorder equations of motion - violation of the NEC generally implies the existence of an instability toward the formation of gradients. In the case of configurations that possess at least some rotational symmetry (e.g., those of interest in cosmology), violation of the NEC always implies instability (Sec. IIC). In cases with less symmetry, it is possible for NEC-violating models to be stable if they exhibit superluminal excitations [6]; however, such models are not causal 7]. In the appendix we show that in any causal scalar model, NEC violation implies instability.

These results can be generalized to quantum theories, including fermions. Analogous results for perfect fluids relate violation of the NEC to either a complex speed of sound or a clumping instability.

\section{Acknowledgments}

The authors thank Alejandro Jenkins for very useful comments. This work was supported by the Department of Energy under DE-FG06-85ER40224.

\section{APPENDIX: CAUSALITY AND STABILITY}

In this appendix we impose an additional causality constraint, which excludes superluminal excitations of the type considered in Refs. [6], 7]. We show that a causal, NEC-violating model cannot be stable. For simplicity, we limit our attention to the case of scalar fields.

\section{Fluctuations}

Consider a theory of $n$ scalar fields $\phi_{a}$ in $d$-dimensional spacetime. Using the first derivative of the field, $\phi_{a \mu}=$ $\nabla_{\mu} \phi_{a}$, we can construct a Lorentz-invariant matrix $X_{a b}=g^{\mu \nu} \phi_{a \mu} \phi_{b \nu}$. The most general Lorentz invariant Lagrangian resulting in the equation of motion of at most second order is $\mathcal{L}=F\left(X_{a b}, \phi_{c}\right)$, where the function $F$ is a Lorentz scalar, but in general it is not a scalar in the flavor space. The second fluctuation of the Lagrangian is

$$
\delta^{2} \mathcal{L}=F^{a \mu b \nu} \delta \phi_{a \mu} \delta \phi_{b \nu}+\cdots
$$

where

$$
F^{a \mu b \nu}=F_{X_{a b}} g^{\mu \nu}+2 F_{X_{a c} X_{b d}} \phi_{c}^{\mu} \phi_{d}^{\nu} .
$$

The equation of motion for the fluctuations is

$$
F^{a \mu b \nu} \delta \phi_{b \mu \nu}+\cdots=0 .
$$


In Eq. A.1 , ellipses denote terms at most linear in the derivatives of fluctuations; such terms are not important for the present analysis. Specifically, they do not enter into the equation for the characteristic surfaces or the dispersion relation for short wavelength fluctuations. See, e.g., Ref. 17] for more on characteristics in partial differential equations. In the case of the dispersion relation, the short wavelength approximation dictates that only terms with the largest number of derivatives matter.

\section{Hyperbolicity and causality}

When the equation of motion is hyperbolic, its domain of influence is bounded by the outside characteristics originating from the region where nontrivial initial data is prescribed. The resulting characteristic surface is given by $\xi(x)=0$, where $\xi_{\mu}=\nabla_{\mu} \xi$ satisfies the characteristic equation

$$
\left\|F^{a \mu b \nu} \xi_{\mu} \xi_{\nu}\right\|=0
$$

and $\|\cdots\|$ denotes a determinant in flavor space. For a hyperbolic model, characteristics and therefore $\xi_{\mu}$ are real. For such a model, the characteristic surface is inside the light cone $\xi_{0}^{2}-\delta^{i j} \xi_{i} \xi_{j}<0$ (i.e., the model is causal), if and only if the matrix $F^{a \mu b \nu} n_{\mu} n_{\nu}$ is sign-definite for all null $n_{\mu}$. By writing $F^{a \mu b \nu}=\sum_{c} F_{c}^{\mu \nu} v_{c}^{a} v_{c}^{b}$, where $F_{c}^{\mu \nu}$ and $v_{c}^{a}$ are eigenvalues and eigenvectors, respectively, this result is obtained by noticing that the characteristic surface consists of the null surfaces for the metrics $F_{c}^{\mu \nu}$. Each of these null surfaces has to be inside the light cone, hence the above result. From Eq. A.2, this means that the matrix $C^{a b}=2 F_{X_{a c} X_{b d}}\left(\phi_{c}^{\mu} n_{\mu}\right)\left(\phi_{d}^{\nu} n_{\nu}\right)$ is sign-definite.

In terms of $A^{a b}=F_{X_{a b}}, B^{a b}=2 F_{X_{a c} X_{b d}}\left(\phi_{c}^{\mu} \xi_{\mu}\right)\left(\phi_{d}^{\nu} \xi_{\nu}\right)$, and $\omega=-\xi_{\mu} \xi^{\mu}$, the characteristic equation is

$$
\|B-\omega A\|=0 .
$$

For nonsingular $A$, the parameter $\omega$ is equal to one of the eigenvalues of the matrix $A^{-1} B$. Causality requires $\omega>0$ and so $^{2} A^{-1} B>0$.

Consider a Lorentz boost

$$
\phi_{a}^{\mu} \xi_{\mu} \rightarrow \tilde{\phi}_{a}^{\mu} \tilde{\xi}_{\mu} \approx \tilde{\phi}_{a}^{\mu} n_{\mu}
$$

for some particular null vector $n_{\mu}$. (It is easy to check that $\tilde{\xi}_{\mu}$ can always be made nearly null by appropriate choice of frame.) In this new frame, $B^{a b}$ is arbitrarily close to the matrix $C^{a b}$ evaluated on this particular $n_{\mu}$. Then, because $C$ is sign-definite for all $n_{\mu}$, the signdefiniteness of $B$ follows. This means that if a model is causal, then either $A>0, B>0$ or $A<0, B<0$. This result can be extended to singular $A$ as well.

\footnotetext{
${ }^{2}$ The inequality $Q>0(Q<0)$ for the matrix $Q$ means that $Q$ is positive (negative) definite.
}

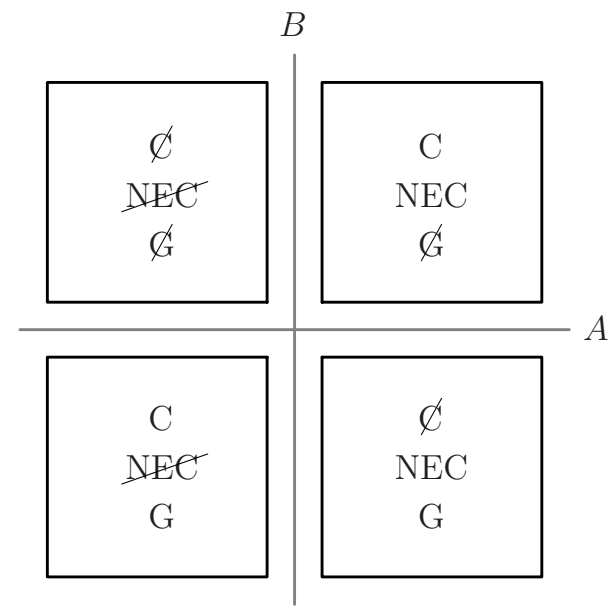

FIG. 5: The NEC, causality (here C), and quantum mechanical stability (the absence of ghosts, here $G$ ) are all determined by the sign-definiteness of matrices $A$ and $B$. The NEC holds for $A>0$; the model is causal for $A^{-1} B>0$ and ghost-free for $B>0$. A model is ghost-free and causal only if it obeys the NEC.

\section{Stability and NEC}

To study local stability, it is enough to consider the equation of motion in a small neighborhood of a given point $x$. In such a neighborhood, we can ignore spacetime dependence of $F^{a \mu b \nu}$ and solve the equation in terms of Fourier modes with momenta $k_{\mu}$. Also, in the short wavelength approximation, only terms quadratic in derivatives matter. The corresponding dispersion relation is

$$
\left\|F^{a \mu b \nu} k_{\mu} k_{\nu}\right\|=0 .
$$

Classical stability requires that $k_{\mu}$ is real, which means that the matrix $F^{a \mu b \nu}$ satisfies the same condition which is required for the characteristics to be real. In other words, models are classically stable if and only if they are hyperbolic. To avoid ghosts, i.e, quantum mechanical instabilities 11, there is an additional condition that the matrix $F^{a 0 b 0}$ be positive definite. Except in the trivial case where $F_{X_{a b} X_{c d}}$ vanishes, we can always choose such a Lorentz boost that $F^{a 0 b 0}$ is arbitrarily close to $C^{a b}$ for some $n_{\mu}$. Since $B$ and $C$ are of the same sign definiteness, the matrix $F^{a 0 b 0}$ can be positive definite, and therefore the model is ghost-free, only if $B>0$. Then, from the previous section, the combined requirement that the model be both causal and ghost-free can be satisfied only if $A>0$ and $B>0$.

From the energy-momentum tensor

$$
T_{\mu \nu}=-F g_{\mu \nu}+2 F_{X_{a b}} \phi_{a \mu} \phi_{b \nu},
$$

it follows that the NEC is equivalent to $A \geq 0$. Thus, for a hyperbolic, causal $\left(A^{-1} B>0\right)$, classically and quantum mechanically stable model $(B>0)$, the NEC is satisfied $(A \geq 0)$. Vice versa, a causal, hyperbolic (and 
therefore classically stable), NEC-violating model cannot be quantum mechanically stable. See Fig. 5 for a diagram summarizing the relation between quantum mechanical stability, causality, and the NEC.

\section{Example}

For the case $n=1$, combining Eqs. A.2 and A.4, the characteristic equation is

$$
F_{X} \xi_{\mu} \xi^{\mu}+2 F_{X X}\left(\phi^{\mu} \xi_{\mu}\right)^{2}=0
$$

and the model is causal if $F_{X}^{-1} F_{X X}>0$. Violation of the NEC and absence of ghost instability imply $F_{X}<0$ and $F_{X X}>0$, respectively. We see that $\mathrm{NEC}$ violation and the absence of ghost instability imply non-causal behavior for this model. Moreover, for $F_{X}<0$ and $F_{X X}>0$ it is impossible to find a Lorentz frame in which the Hamiltonian

$$
\begin{aligned}
\delta^{2} \tilde{\mathcal{H}} & =\left[F_{X}+2 F_{X X}\left(\tilde{\phi}^{0}\right)^{2}\right]\left(\delta \tilde{\phi}_{0}\right)^{2} \\
& +\left[F_{X} \delta^{i j}-2 F_{X X} \tilde{\phi}^{i} \tilde{\phi}^{j}\right] \delta \tilde{\phi}_{i} \delta \tilde{\phi}_{j}+\cdots(
\end{aligned}
$$

is positive definite since the second term is always negative. Clearly, this can happen only for a non-causal model. When superluminal modes are present (i.e., a model is not causal), the sign of the Hamiltonian can be changed by performing a Lorentz boost.
[1] R. V. Buniy and S. D. H. Hsu, Phys. Lett. B 632, 543 (2006) arXiv:hep-th/0502203.

[2] For discussion of the NEC and possible violations, see, e.g., M. Visser, Lorentzian wormholes: From Einstein to Hawking, AIP, Woodbury USA, 1995; E. E. Flanagan and R. M. Wald, Phys. Rev. D 54, 6233 (1996); J. Fewster and T. A. Roman, Phys. Rev. D 67, 044003 (2003); L. H. Ford, Int. J. Theor. Phys. 42, 1219 (2003).

[3] S. W. Hawking and G. F. R. Ellis, The Large Scale Structure of space-time, Cambridge University Press, Cambridge, 1973.

[4] R. Bousso, Rev. Mod. Phys. 74, 825 (2002).

[5] M. S. Morris and K. S. Thorne, Am. J. Phys. 56, 395 (1988); J. L. Friedman, K. Schleich and D. M. Witt, Phys. Rev. Lett. 71, 1486 (1993) [Erratum-ibid. 75, 1872 (1995)]; D. Hochberg and M. Visser, Phys. Rev. D 56, 4745 (1997); M. Visser and D. Hochberg, arXiv:gr-qc/9710001 R. V. Buniy and S. D. H. Hsu, hep-th/0504003 to appear in Phys. Lett. B.

[6] S. Dubovsky, T. Gregoire, A. Nicolis and R. Rattazzi, arXiv:hep-th/0512260

[7] A. Adams, N. Arkani-Hamed, S. Dubovsky, A. Nicolis and R. Rattazzi, arXiv:hep-th/0602178

[8] S. D. H. Hsu, A. Jenkins and M. B. Wise, Phys. Lett. B 597, 270 (2004).

[9] C. Armendariz-Picon, T. Damour and V. Mukhanov, Phys. Lett. B 458, 209 (1999); C. Armendariz-Picon, V. Mukhanov and P. J. Steinhardt, Phys. Rev. Lett. 85, 4438 (2000); C. Armendariz-Picon, V. Mukhanov and
P. J. Steinhardt, Phys. Rev. D 63, 103510 (2001).

[10] A. Sen, JHEP 0204, 048 (2002).

[11] R. R. Caldwell, Phys. Lett. B 545, 23 (2002); S. M. Carroll, M. Hoffman and M. Trodden, Phys. Rev. D 68, 023509 (2003); J. M. Cline, S. Jeon and G. D. Moore, Phys. Rev. D 70, 043543 (2004)

[12] In some curved spacetimes there may not be a welldefined ground state. In de Sitter space, quantum corrections can cause violation of the NEC: V. K. Onemli and R. P. Woodard, Class. Quant. Grav. 19, 4607 (2002); Phys. Rev. D 70, 107301 (2004).

[13] L. Alvarez-Gaume and P. H. Ginsparg, Nucl. Phys. B 243, 449 (1984); L. Alvarez-Gaume and P. H. Ginsparg, Annals Phys. 161, 423 (1985) [Erratum-ibid. 171, 233 (1986)].

[14] R. Jackiw, V. P. Nair, S. Y. Pi and A. P. Polychronakos, J. Phys. A 37, R327 (2004).

[15] For discussion of negative compressibility and mechanical instability, see, e.g., P. M. Chaikin and T. C. Lubensky, Principles of Condensed Matter Physics, Cambridge University Press, 2000; J. L. Barrat and J. P. Hansen, Basic Concepts for Simple and Complex Liquids, Cambridge University Press, 2003; M. D. Sturge, Statistical and Thermal Physics, A. K. Press, Ltd., 2003.

[16] See, e.g., R. Shankar, Principles of Quantum Mechanics, Plenum Press, 1994.

[17] I. G. Petrovskii, Partial Differential Equations, W. B. Saunders Company, Philadelphia, 1967. 